\title{
Lithium-ion Battery Degradation Assessment and Remaining Useful Life Prediction in Hybrid Electric Vehicle
}

\author{
Nabil LAAYOUJ *, Hicham JAMOULI * \\ * LGII Laboratory, National School of Applied Sciences, Ibn Zohr University, \\ Agadir, Morocco \\ E-mail: nabil.laayouj@gmail.com
}

\begin{abstract}
Prognostic activity deals with prediction of the remaining useful life (RUL) of physical systems, based on their actual health state and their usage conditions. RUL prediction gives operators a potent tool in decision making by quantifying how much time is left until functionality is lost. In addition, it can be used to improve the characterization of the material properties, that govern damage propagation for the monitored structure. RUL can be predicted by using three main approaches, namely model-based, datadriven and hybrid approaches. Prognostic methods used later in this paper are hybrid and data-driven approaches, which employ the Particle Filter in the first one and the autoregressive integrated moving average in the second. The performance of the suggested approaches is evaluated in a comparative study on data collected from lithium-ion battery of hybrid electric vehicle.
\end{abstract}

Keywords - Remaining useful life; Prognosis; Particle Filter; ARIMA.

\section{INTRODUCTION}

Prognosis and health management (PHM) [1] will have significant impact on increasing safety as well as reducing operating and maintenance costs. This can be achieved by providing an accurate quantification of degradation and damage at an early stage to reduce or eliminate malfunctions. Furthermore, PHM consists of three main routines: fault detection, diagnosis and prognosis. A prognosis has recently attracted a lot of research interest due to the need of models for accurate RUL prediction [2].

Numerous methods and tools can be employed to evaluate the size of damage by predicting the RUL value. Prognosis techniques can be categorized under three approaches based on the usage of information: model-based, data-driven and hybrid approaches. The model based approach [3], assumes that a model of system behavior is available and uses this model to predict the future of the system behavior. Some recent developments in the model-based approach have been reported in the literature. Such as lumped parameter model [4], functional models [5] and first principal models [6].

The data-driven approach [7] aims at transforming the data provided by sensors into relevant models. In the literature, there are the following works: relevance vector machine [8] and neural network [9]. The hybrid approach [10] combines the two approaches cited earlier and includes Bayesian techniques [11][12].

In this work, the researchers will study two main approaches to predict RUL. The first approach is a hybrid prognosis using a Particle Filter method, which employs both, state dynamic model and a measurement data. The second approach is a datadriven prognosis based on routinely collected data, using autoregressive integrated moving average (ARIMA) model to estimate the system degradation.

The application of Particle Filters to prognosis has been reported in the literature. For example, prediction of lithium-ion battery capacity depletion [13], degradation prediction of a thermal processing unit in semiconductor manufacturing [14], and remaining useful life prediction of a mechanical component subject to fatigue crack growth [15]. The reported application results have shown that Particle Filters represent a potentially powerful prognosis tool due to its capability in handling non-linear dynamic systems and non-Gaussian noises, using efficient sequential importance sampling [16][17] to approximate the future state probability distributions.

Many works around ARIMA model have been developed. Among these are mechanical deterioration prognosis [18] [19] and economic forecasts [20][21]. In the present study, the researchers will use the ARIMA model to predict 
degradation by computing RUL.

This paper is organized as follows: section 2 contains the descriptions of the approaches at the basis of RUL prediction; in section 3, the results of the application of the methods are presented, and an evaluation of their performance is given; and finally, some conclusions on the advantages and limitations of the approaches are given in section 4 .

\section{SUGGESTED APPROACHES BASED ON THE RUL PREDICTION}

\section{A. Prognosis of degradation and remaining useful life}

The term prognosis is originally used in medicine for the prediction of a course of an illness. But, later on it has been introduced to industry to predict the future operating status of equipments, and to set an efficient treatment.

The practitioner uses the results of the forecast models to determine the most appropriate treatment. These forecast models based on simple mathematical tools (e.g. decision tree, conditional probability) [22], or on more sophisticated (e.g. Markov processes, neural networks, and genetic algorithms) [23].

As mentioned earlier, the main objective of prognosis is to determine the time before failure. Note that the data prognosis is important information that may be used in the decision process. For example, this data prognosis can be used to delay the maintenance interventions, or stop a machine before its future maintenance due to earlier default.

\section{B. Methods of prognosis}

\section{Particle filter for prognosis}

The Particle Filter method [24] is a Monte Carlo technique for the solution of the state estimation problem. The key idea is to represent the required posterior density function by a set of random samples (particles) with associated weights, and to compute the estimations based on these samples and weights. As the number of samples becomes very large, this Monte Carlo characterization becomes an equivalent representation of the posterior probability function, and the solution approaches the optimal Bayesian estimation.

\section{Particle filter model}

Consider the dynamic system described by the following discrete time model [15]:

$$
\begin{aligned}
& x_{k}=f_{k}\left(x_{k-1}, v_{k}\right) \\
& z_{k}=h_{k}\left(x_{k}, \omega_{k}\right)
\end{aligned}
$$

\section{Where:}

$f_{k}$ : is the state transition function (damage model)

$v_{k}$ : is state noise vector of known distribution

$h_{k}:$ is the measurement function

$\omega_{k}:$ is the measurement noise vector

The goal of tracking is to recursively estimate $x_{k}$ by using the set of all available measurements $z_{1: k}=\left(z_{1}, \ldots, z_{k}\right)$ up to time $\mathrm{k}$, and to create a conditional state PDF (probability density function). Like any Bayesian estimation, two steps are employed: prediction and update.

In the prediction step, the researchers consider that the PDF $p\left\langle x_{k-1} \mid z_{1: k-1}\right\rangle$ previous state estimate at time $k-1$ and the process model (1), both are used to obtain the prior PDF of the state at time $k$ as shown in Chapman-Kolmogorov equation.

$$
p\left\langle x_{k} \mid z_{1: k-1}\right\rangle=\int p\left\langle x_{k} \mid x_{k-1}\right\rangle p\left\langle x_{k-1} \mid z_{1: k-1}\right\rangle d x_{k-1}
$$

In the update step, at time, a measurement becomes available (from the likelihood function defined by the measurement model (2)), and this may be used to update the prior distribution to generate the posterior state PDF via Bayes rule (4).

$p\left\langle x_{k} \mid z_{1: k}\right\rangle=\frac{p\left\langle z_{k} \mid x_{k}\right\rangle p\left\langle x_{k} \mid z_{1: k-1}\right\rangle}{p\left\langle z_{k} \mid z_{1: k-1}\right\rangle}$

In order to obtain exact state estimation solutions for Equations (3) and (4), the actual distributions are approximated by a set of samples and their normalized weights. Consider $\left\{x_{0: k}^{i}, w_{k}^{i}\right\}_{i=1}^{N_{S}}$ a random measure that characterizes the posterior PDF 
$p\left\langle x_{k} \mid z_{1: k}\right\rangle$, where $x_{0: k}^{i}$ and $w_{k}^{i}$ are, respectively a set of support points and associated weights. The weights are normalized such that $\sum_{i} w_{k}^{i}=1$. Then, the posterior density at time $k$ is approximated [24] as

$$
p\left\langle x_{k} \mid z_{1: k}\right\rangle \approx \sum_{i=1}^{N_{S}} w_{k}^{i} \delta\left(x_{0: k}-x_{0: k}^{i}\right)
$$

The weight process based on importance sampling [24], such that the weight update equation is given by

$$
w_{k}^{i} \propto w_{k-1}^{i} \frac{p\left\langle z_{k} \mid x_{k}^{i}\right\rangle p\left\langle x_{k}^{i} \mid x_{k-1}^{i}\right\rangle}{\pi\left\langle x_{k}^{i} \mid x_{0: k-1}^{i} z_{1: k}\right\rangle}
$$

When $N_{S} \rightarrow \infty$ the importance density function $\pi\left\langle x_{k} \mid x_{0: k-1} z_{1: k}\right\rangle$ can be approximated by the prior PDF $p\left\langle x_{k} \mid x_{k-1}\right\rangle$, and weight becomes [16]

$$
w_{k}^{i} \propto w_{k-1}^{i} p\left\langle z_{k} \mid x_{k}^{i}\right\rangle
$$

Another problem arises is the Degeneracy phenomenon, where after a few iterations, all but one particle will have negligible weight. This degeneracy explains that a large number of updating particles is around zero. To overcome this problem, considering resampling procedure [25] at each step, the researchers assign $w_{k-1}=\frac{1}{N_{S}}$ for all the particle weights so they have

$$
w_{k}^{i} \propto p\left\langle z_{k} \mid x_{k}^{i}\right\rangle
$$

To implement SIR (sequential importance resampling) filter, as in (7) and (8), the researchers need to know process model, measurement model and likelihood function $p\left\langle z_{k} \mid x_{k}\right\rangle$

\section{RUL Prediction using Particle Filter}

Once the estimated parameter is obtained, the future damage state and RUL can be predicted by progressing the damage state until it reaches the threshold (Fig. 1). The PDF curve represents the progress of damage state until it reaches the threshold. The distribution of RUL can be obtained by subtracting this PDF from the threshold

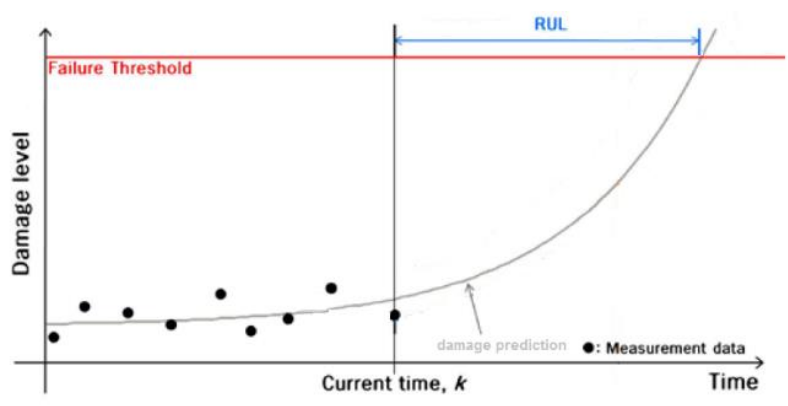

Fig .1. Illustration of RUL calculation

\section{ARIMA model for prognosis}

One of the important and widely used time series model is the autoregressive integrated moving average (ARIMA) model, which is a generalization of ARMA model. It requires only the historical time series data. These models are fitted to time series data to predict future points in the series (forecasting).

\section{ARIMA forecasting method}

ARIMA is a forecasting technique, noted as ARIMA $(p, d, q)$, the general model was introduced by Box and Jenkins [26]. It is a method which allows both autoregressive (AR) and moving average (MA). It explicitly includes differencing in the formulation of the model. Where, $p$ and $q$ are, respectively, autoregressive parameter and moving average parameter, while $d$ is the number of non-seasonal differences. The autoregressive part of the model of order $p$ is written:

$x_{t}=c+\sum_{i=1}^{p} \phi_{i} x_{t-i}+\varepsilon_{t}$

Where $x_{t}$ is a stationary series, $x_{t-i}$ represents lag $i$ of $x_{t}$, the $\phi_{i}, i=1, \ldots, p$ are the parameters of the model, $\mathrm{c}$ is a constant and $\varepsilon_{t}$ is a white noise. The moving average part of the model of order $q$ is written:

$$
x_{t}=\mu+\sum_{i=1}^{q} \theta_{i} \varepsilon_{t-i}+\varepsilon_{t}
$$


Where the $\theta_{i}, i=1, \ldots, q$ are the parameters of the model, $\mu$ is the expectation of $x_{t}$, which is often assumed to equal zero, and the $\varepsilon_{t}, \varepsilon_{t-1}, \ldots, \varepsilon_{t-q}$ are white noise error terms. After an initial differencing step (corresponding to the integrated part of the model), the researchers can present the ARIMA $(p, d, q)$ as ARMA $(p, q)$ process:

$x_{t}=c+\varepsilon_{t}+\sum_{i=1}^{p} \phi_{i} x_{t-i}+\sum_{i=1}^{q} \theta_{i} \varepsilon_{t-i}$

The estimation of the ARIMA model corresponding to some learning data is done through Box and Jenkins methods [26]. A procedure of the forecasting can be summarized as:

- Check stationary: If the data are not stationary, they need to be transformed into stationary data using the differencing technique.

- Identification: to specify the appropriate number of autoregressive term $p$, and moving average term $q$ from the autocorrelation function (acf) and partial autocorrelation function (pacf) correlograms.

- Forecasting: Based on the forecasting model, multi-step-ahead prediction is then conducted to forecast the final failure time.

- Verification: If the predictions result in an unexpected trend, repeat steps 2 and 3 until the model fits the historical data appropriately.

\section{RUL prediction using ARIMA}

Prediction of lifetime using ARIMA can be expressed in two parts: construction of model and prediction of state. In the first step, the researchers construct the corresponding coefficients (differencing, autoregressive and moving average terms) and they obtain an ARIMA model. Next step, when the model is built, a sample is selected to be estimated. Remaining Useful Life (RUL) is defined as the number of predictions from current state until the failure states reached the threshold. The following Fig. 2 shows the forecasting method for RUL prediction.

\section{Hybrid electric vehicle (HEV)}

An electric car is powered by an electric motor instead of a gasoline or diesel engine. The electric car (also known as electric vehicle EV) uses energy stored in its battery (or series of batteries), which are recharged by different sources. There is a variety of electric vehicles available in the world, among these HEV (hybrid electric vehicle), the PHEV (plug-in hybrid electric vehicle) and BEV (battery electric vehicle).

The researchers have oriented their work towards the HEV (Fig. 3), where a small battery is placed on board, and when the vehicle brakes, the energy is stored in the battery and it can later be used to power the electric motor which assists the gasoline engine. HEV typically provides better fuel economy than similar conventional vehicles. This is why it is necessary to develop a prognosis of battery degradation, in order to ensure a proper functionality.

The cell (or battery) studied in this work [27] is a lithium-ion with $1 \mathrm{Ah}$ capacity and $3.75 \mathrm{~V}$. The cell is part of a battery pack (Battery pack $=15$ cells) which is used to collect and distribute electric power (direct current power), mainly to the electrical drive. The researchers use the $25^{\circ} \mathrm{C}$ as the baseline for measurement. A simple form of the empirical degradation model is expressed by an exponential growth model as follows [28]

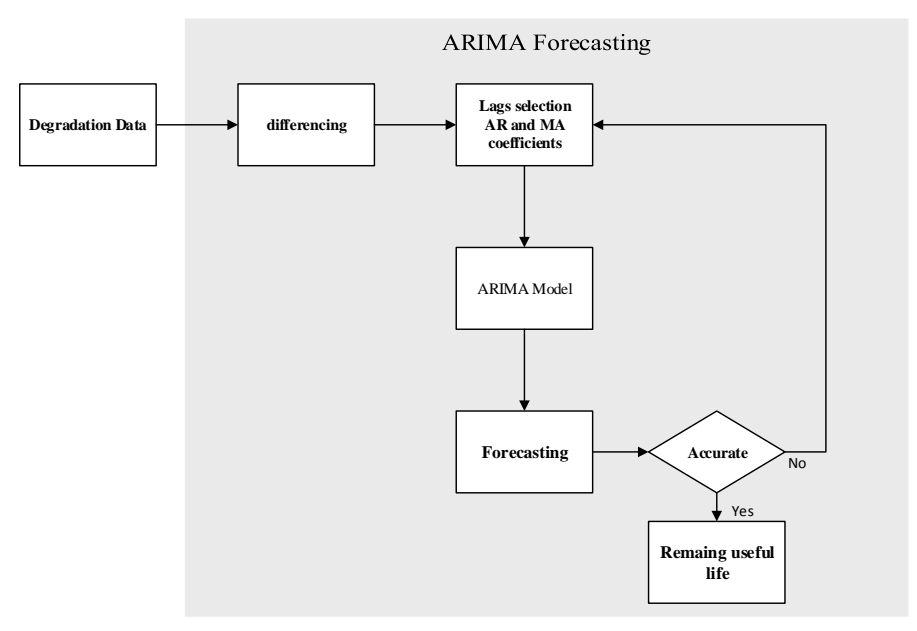

Fig .2. ARIMA prediction Algorithm

$$
\lambda=a \exp (-b t)
$$

Where $\mathrm{a}, \mathrm{b}$ are model parameters, $\mathrm{t}$ is the time, and $\lambda$ is internal cell performance. The equivalent electrical model is introduced as shown in Fig. 4. where, $C_{D L}$ is the double layer capacitance, $R_{C T}$ is 
the charge transfer resistance, $R_{W}$ is the Warburg impedance and $R_{E}$ is the electrolyte resistance. The internal cell performance is observed instead of the capacity. Additionally, there is a relationship between $R_{E}+R_{C T}$ and $C / 1$ capacity; $R_{E}+R_{C T}$ is inversely proportional to the $C / 1[28]$. Also, the observed data are assumed to be given as a $C / 1$ capacity. $R_{W}$ and $C_{D L}$ showed negligible change over the ageing process and are excluded from further analysis. The threshold for fault declaration has arbitrarily been chosen.

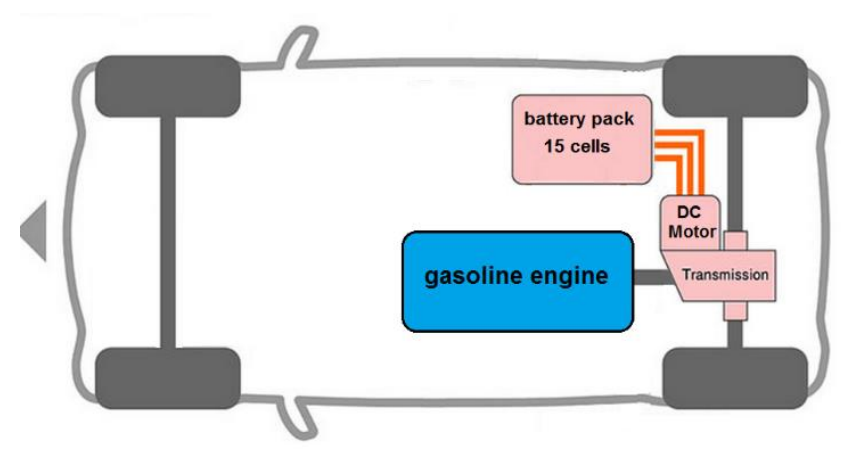

Fig .3. Plan of hybrid electric vehicle

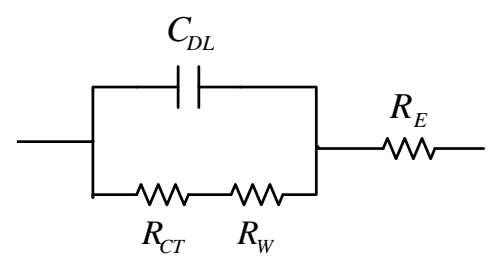

Fig .4. Equivalent circuit diagram of a Li-ion Cell

Table 1. Data of Degradation

\begin{tabular}{|c|c|c|c|c|c|}
\hline Time (T) & Intial,1 & 2 & 3 & 4 & 5 \\
\hline C/1 (Ah) & 1,000 & 0.981 & 0.859 & 0.811 & 0.788 \\
\hline Time(T) & 6 & 7 & 8 & 9 & 10 \\
\hline C/1 (Ah) & 0.714 & 0.680 & 0.612 & 0.56 & 0.568 \\
\hline
\end{tabular}

\section{RUL prediction and results}

The Particle Filter uses the exponential growth model (12) to obtain the prior PDF (1). The measurement and process noise variance $\omega_{k}$ and $v_{k}$ respectively were modeled as Gaussian densities. In the Particle Filter, $a$ and $b$ are incorporated as internal cell parameters $R_{E}$ and $R_{C T}$. The values of $a$ and $b$ in the actual state are used as initial estimations. Then, the resampling of particles is applied to each iteration to solve the degeneracy problem. The predictions are progressed until they reach the threshold to get the RUL. The failure threshold is defined when the capacity fades by $35 \%$.

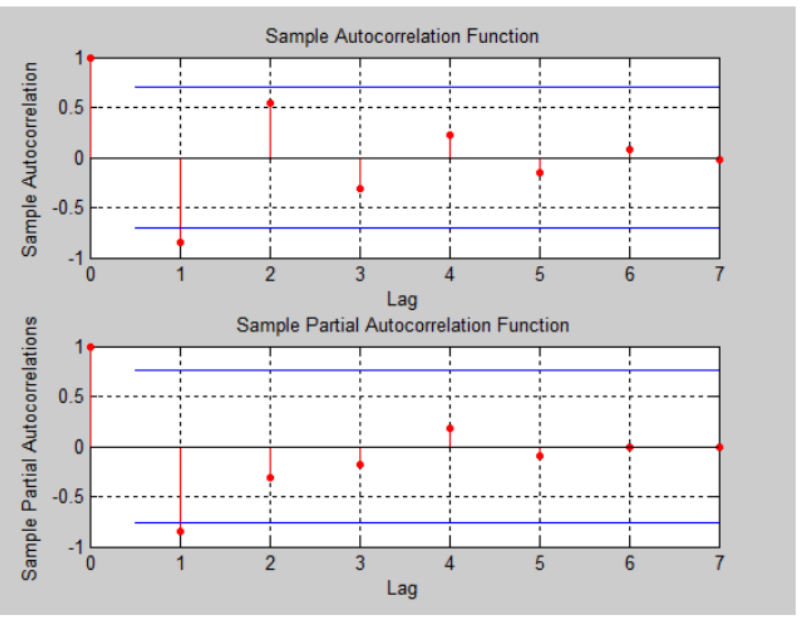

Fig .5. Autocorrelation and partial autocorrelation plots for ARIMA model

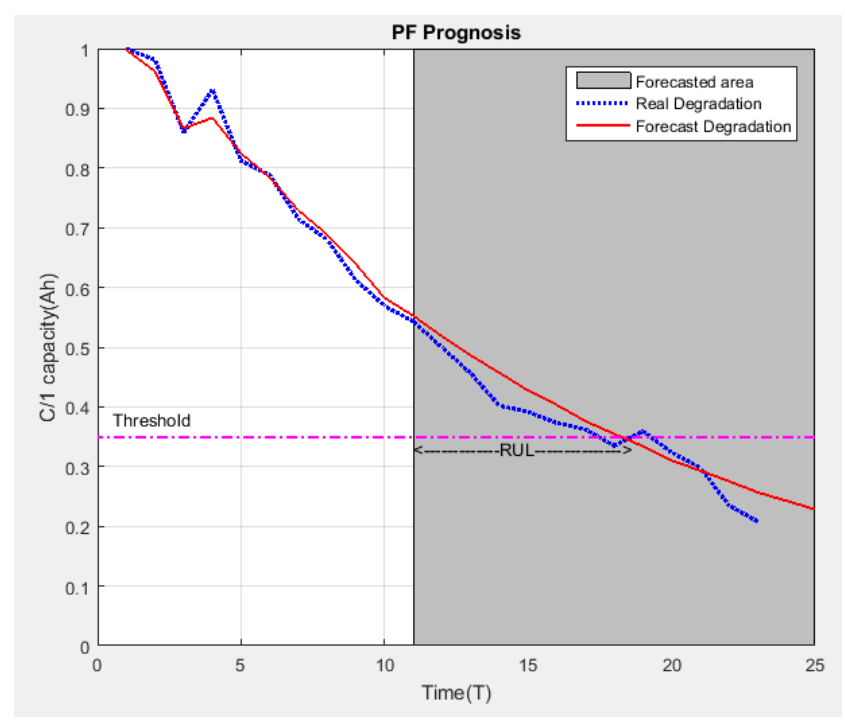

Fig .6. Trajectory of degradation for the cell of battery pack using Particle Filter

ARIMA (p,d,q) model uses simply the capacity data measurement to predict the future degradation. In our case, the data are roughly exponential in nature; $d$ is 
chosen to be 2 in order to remove the non-stationarity, both $p$ and $q$ are chosen to be 1 (Fig. 5). The capacity data measurement at every 100 charge/discharge cycles are given in Table 1, where $1 \mathrm{~T}=100$ charge/discharge cycles.

Fig. 6 shows the real and the estimated values of the cell degradation using Particle Filter. Although the predicted results are close to the real values throughout the prediction area. In Fig. 7, the estimated data of the cell degradation obtained by ARIMA model are far off the real values after $T=15$.

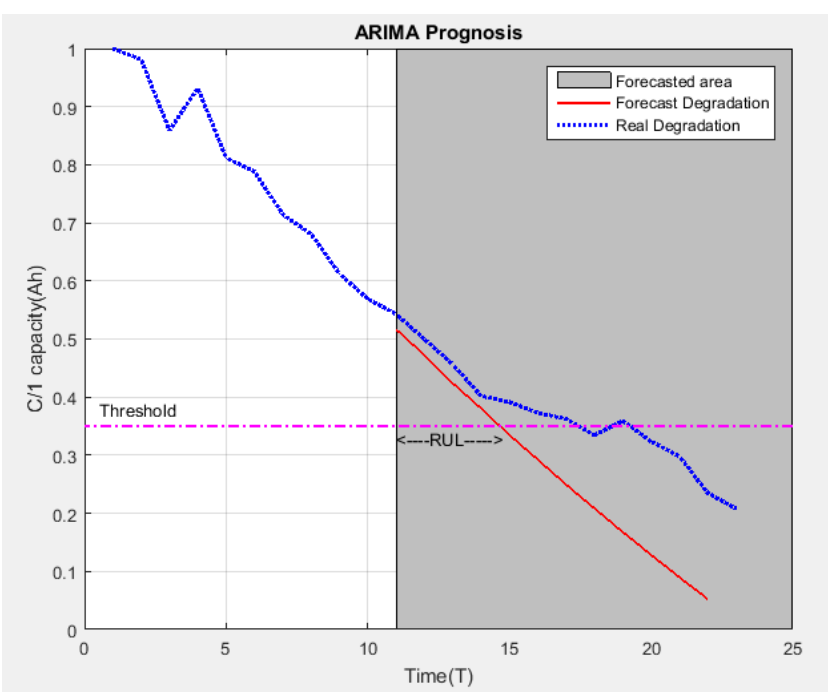

Fig .7. Trajectory of degradation for the cell of battery pack using ARIMA model

The performance of both methods has been evaluated by the Mean Absolute Scaled Error (MASE) [29] and Root Mean Square Error (RMSE).

Definition of MASE:

A scaled error is defined as

$$
q_{k}=\frac{Y_{k}-\hat{Y}_{k}}{\frac{1}{N-1} \sum_{i=2}^{N}\left|Y_{i}-Y_{i-1}\right|}
$$

Where $Y$ is the real value of testing cell, $\hat{Y}$ is the prediction value and $\mathrm{N}$ is the number of prediction data set. The MASE is simply

$$
\operatorname{MASE}=\operatorname{mean}\left(\left|q_{k}\right|\right)
$$

$$
R M S E=\sqrt{\frac{1}{N} \sum_{t=1}^{N}[Y-\hat{Y}]^{2}}
$$

The detail results are shown in Table 2 .

Table 2. MASE and RMSE measurement of the cell prognosis

\begin{tabular}{|r|c|c|}
\hline $\begin{array}{c}\text { predictive } \\
\text { approach }\end{array}$ & Particle Filter & ARIMA model \\
\hline MASE & 0,7345 & 2,6163 \\
\hline RMSE & 0,0253 & 0,0719 \\
\hline
\end{tabular}

From Table 2, one can find that the calculation of MASE and RMSE for ARIMA model is superior to the Particle Filter. Consequently, the Particle Filter is more accurate than the ARIMA model. In this study, the researchers have illustrated how the Particle Filter method can provide accurate predictions of the RUL, as presented in [28][30] over conventional data-driven methods without physical model.

\section{CONCLUSION}

In this study, the researchers interested here in two approaches for health prognostics using Particle Filter and ARIMA model. The goal in applying these methods is to calculate RUL. In addition, the RUL gives the best idea about the degradation of each system. The results showed that the Particle Filter was more faithful to the simulated data. The researchers considered different frequencies of inspection for the measurement.

This study highlights the value of having a physical failure model to improve the accuracy of results. In contrast, the ARIMA needs great possible historical data in order to give proper results without the need for physical model. However, the disadvantage of the Particle Filter compared to ARIMA model is the degradation model requirement, which is not always easy in the case of a large-scale system. Remember that both approaches require a knowledge of a threshold corresponding to the physical system failure to plan the actions of preventive maintenance, and expect to benefit from the opportunistic maintenance. Finally, the obtained results in this paper show that the Particle Filter is more efficient than the ARIMA model.

Definition of RMSE: 


\section{REFERENCES}

[1] K. Medjaher, Tobon-Mejia and N. Zerhouni, "Remaining useful life estimation of ceritical components with application to bearings beliability", IEEE Transactions on, vol.61, no.2, 2012, pp.292-302.

[2] A. Mosallam, K. Medjaher and N. Zerhouni, "Bayesian approach for remaining useful life prediction", chemical engineering transactions, vol. 33, 2013, pp. 139-144.

[3] A. Heng, A. C. Tan, J. Mathew, N. Montgomery, D. Banjevic, and A. K. Jardine, "Intelligent condition-based prediction of machinery reliability", Mechanical Systems and Signal Processing, vol. 23, no. 5, 2009, pp. 1600-1614.

[4] C. J. and H. Lee, "Gear fatigue crack prognosis using embedded model, gear dynamic model and fracture mechanics", Mechanical systems and signal processing, vol. 19, 2005, pp. 836846.

[5] P. Toguyeni, A. K. A and E. Craye "Structural and functional approach for dependability in FMS", IEEE International Conference on Systems, Man, and Cybernetics, 1999.

[6] Jaw, J.C, Inc, S. M. and Tempe, A. Z., "Neural networks for model-based prognostics", IEEE Aerospace Conference, 1999.

[7] M. Dong and D. He, "A Segmental Hidden SemiMarkov Model (HSMM)-based diagnostics and prognostics framework and methodology", Mechanical Systems and Signal Processing, vol. 21, no. 5, 2007, pp. 2248-2266.

[8] M. Tipping, the Relevance Vector Machine. In Advances in Neural Information Processing Systems, MIT Press, Cambridge, 2000.

[9] A.N. Srivastava and S. Das, "Detection and prognostics on low dimensional systems", IEEE Transactions on Systems, Man, and Cybernetics, Part C: Applications and Reviews, vol. 39, 2009, pp. 44-54.

[10] J. Yan and J. Lee, "A hybrid method for on-line performance assessment and life prediction in drilling operations", IEEE International Conference on Automation and Logistics, 2007.

[11] J. W .Sheppard, M. A. Kaufman, A. Inc and M. D. Annapolis, "Bayesian diagnosis and prognosis using instrument uncertainty", IEEE Autotestcon, 2005, pp. 417423.

[12] B. Saha and K. Goebel, "Uncertainty management for diagnostics and prognostics of batteries using Bayesian techniques", IEEE Aerospace Conference, 2008.

[13] B. Saha, K. Goebel, S. Poll, and J. Christophersen, "Prognostics methods for battery health monitoring using a Bayesian framework", IEEE Transactions on Instrumentation and Measurement, vol. 58, no. 2, 2009, pp. 291-296.

[14] S. Butler and J. Ringwood, "Particle Filters for remaining useful life estimation of abatement equipment used in semiconductor manufacturing", Proceedings of the First Conference on Control and Fault-Tolerant Systems, Nice, France, 2010, pp. 436 - 441.

[15] E. Zio and G. Peloni, "Particle filtering prognostic estimation of the remaining useful life of nonlinear Components", Reliability Engineering and System Safety, vol.96, 2011, pp. 403- 409.

[16] B. Ristic, S. Arulampalam, N. Gordon, Beyond the Kalman Filter, Artech House, Boston, 2004.

[17] A. Kong, J.S. Liu and W.H. Wong, "Sequential imputations and Bayesian missing data problems", Journal of the American Statistical Association, vol. 89, no. 425, 1993, pp. 278-288.

[18] P. Kosasih, W. Caesarendra, A. K.Tieu, A. Widodo \& Moodie, C. A. S, "Degradation trend estimation and prognosis of large low speed slewing bearing lifetime", Applied Mechanics and Materials, 493, 2014, pp. 343-348.

[19] A. Guclu1, H. Yılboga1, O. F. Eker, F. Camci, I. Jennions, "Prognostics with autoregressive moving average for railway turnouts", Annual Conference of the Prognostics and Health Management Society, 2010. 
[20] G. Peter Zhang, "Time series forecasting using a hybrid ARIMA and neural network model", Neurocomputing 50, 2003, pp. $159-175$.

[21] M. E. Andreica, N. Cataniciu, M. I. Andreica. "Econometric and neural network analysis of the labor productivity and average gross earnings indices in the romanian industry", Proceedings of the 10thWSEAS International Conference on Mathematics and Computers in Business and Economics, Mar 2009, pp.106-111.

[22] V. Tran, B. Yang, O. MS, A. Tan, "Machine condition prognosis based on decision trees and one-step-ahead prediction", Mechanical System and Signal Processing, vol. 22, 2008, pp. 11791193.

[23] P.J.F. Lucas and A. Abu-Hanna, "Prognostic methods in medicine", Artificial Intelligence in Medicine, vol. 15, 1999, pp.105-119.

[24] S. Arulampalam, S. Maskell, N. Gordon and T. Clapp, "A tutorial on Particle Filters for on-line non-linear/non-Gaussian Bayesian tracking", IEEE Trans. Signal Processing, vol. 50, 2001, pp. 174-188.

[25] N. J. Gordon, D. J. Salmond, A. F. Smith, "Novel approach to nonlinear/non-Gaussian Bayesian state estimation". IEEE Proceedings F (Radar and Signal Pro-cessing) (vol. 140, no. 2), 1993, pp. 107-113.

[26] G.B.P. Box, G.M. Jenkins, G.C. Reinsel, "Time series analysis, forecasting and control", 3rd ed., Prentice Hall,Englewood Cliffs, NJ, 1994.

[27] K. Smith, M. Earleywine, E. Wood, J. Neubauer and A. Pesaran, "Comparison of plug-in hybrid electric vehicle battery life across geographies and drive", SAE international, 2012.

[28] B. Saha1, K. Goebel and J. Christophersen, "Comparison of prognostic algorithms for estimating remaining useful life of batteries", Transactions of the Institute of Measurement \& Control, vol. 31, no. 3-4, 2009, pp. 293-308.

[29] R. J. Hyndman et A. B. Koehler, "Another look at measures of forecast accuracy", International Journal of Forecasting, 22, 2006, pp. 679-688.

[30] D. An, J. Choi and N. Ho Kim, "A tutorial for model-based prognostics algorithms based on matlab code", Annual Conference of Prognostics and Health Management Society, 2012. 